\title{
SIMION'S TYPE $B$ ASSOCIAHEDRON IS A PULLING TRIANGULATION OF THE LEGENDRE POLYTOPE
}

\author{
RICHARD EHRENBORG, GÁBOR HETYEI AND MARGARET READDY
}

\begin{abstract}
We show that Simion's type $B$ associahedron is combinatorially equivalent to a pulling triangulation of a type $B$ root polytope called the Legendre polytope. Furthermore, we show that every pulling triangulation of the Legendre polytope yields a flag complex. Our triangulation refines a decomposition of the Legendre polytope given by Cho. We extend Cho's cyclic group action to the triangulation in such a way that it corresponds to rotating centrally symmetric triangulations of a regular $(2 n+2)$-gon. Finally, we present a bijection between the faces of the Simion's type $B$ associahedron and Delannoy paths.
\end{abstract}

\section{INTRODUCTION}

Root polytopes arising as convex hulls of roots in a root system have become the subject of intensive interest in recent years [1, 8, 10, 13, 17, 18. Another important area where geometry meets combinatorics is the study of noncrossing partitions, associahedra and their generalizations. In this context Simion [20] constructed a type $B$ associahedron whose facets correspond to centrally symmetric triangulations of a regular $(2 n+2)$-gon. Burgiel and Reiner [4] described Simion's construction as providing "the first motivating example for an equivariant generalization of fiber polytopes, that is, polytopal subdivisions which are invariant under symmetry groups". It was recently observed by Cori and Hetyei [9] that the face numbers in this type $B$ associahedron are the same as the face numbers in any pulling triangulation of the boundary of a type $B$ root polytope, called the Legendre polytope in [13].

In this paper we show that the equality of these face numbers is not a mere coincidence. We prove the type $B$ associahedron is combinatorially equivalent to a pulling triangulation of the Legendre polytope $P_{n}$. The convex hull of the positive roots among the vertices of the Legendre polytope and of the origin is a type $A$ root polytope $P_{n}^{+}$. Cho [7] has shown that the Legendre polytope $P_{n}$ may be decomposed into copies of $P_{n}^{+}$that meet only on their boundaries and that there is a $\mathbb{Z}_{n+1}$-action on this decomposition. Our triangulation representing the type $B$ associahedron as a triangulation of the Legendre polytope refines Cho's decomposition in such a way that extends the $\mathbb{Z}_{n+1}$-action to the triangulation. The effect of this $\mathbb{Z}_{n+1}$-action on the centrally symmetric triangulations of the $(2 n+2)$-gon is rotation.

Date: November 25, 2018.

2000 Mathematics Subject Classification. Primary 52B05, 52B12, 52B15, Secondary 05A15, 05 E45. 
Simion observed algebraically that the number of $k$-dimensional faces of the type $B$ associahedron is given by the number of lattices paths between $(0,0)$ and $(2 n, 0)$ taking $k$ up steps $(1,1), k$ down steps $(1,-1)$, and $n-k$ horizontal steps $(2,0)$. Such paths are known as balanced Delannoy paths. In Section 8 we give a combinatorial proof by providing a bijection between the faces of the type $B$ associahedron and Delannoy paths. We give two presentations of this bijection, one recursive and one non-recursive.

Our paper is structured as follows. In the preliminaries we discuss the Simion type $B$ associahedron, the Legendre polytope and pulling triangulations. In Section 3 we show that every pulling triangulation of the Legendre polytope is a flag complex. We introduce an arc representation of Simion's type $B$ diagonals in Section 4 and obtain conditions for when a pair of $B$-diagonals do not cross. A bijection between the set of $B$-diagonals and the vertex set of the Legendre polytope is obtained by the intermediary of our arc representation in Section 5. We characterize when $B$-diagonals cross in terms of crossing and nesting conditions on the arrows associated to the vertices of the Legendre polytope. This characterization is used in Section 6 to define a triangulation of the boundary of the Legendre polytope where each face corresponds to a face in the type $B$ associahedron. Since both complexes are flag and have the same minimal non-faces, we conclude that they are the same polytope. We end this section by describing the facets in our triangulation. In Section 7 we prove that our triangulation refines Cho's decomposition and that his $\mathbb{Z}_{n+1}$-action corresponds to rotating the regular $(2 n+2)$-gon. In Section 8 we present a bijection between faces of the type $B$ associahedron and Delannoy paths.

We end the paper with comments and future research directions.

\section{Preliminaries}

2.1. Simion's type $B$ associahedron. Simion [20] introduced a simplicial complex denoted by $\Gamma_{n}^{B}$ on $n(n+1)$ vertices as follows. Consider a centrally symmetric convex $(2 n+2)$-gon, and label its vertices in the clockwise order with $1,2, \ldots, n, n+1, \overline{1}, \overline{2}, \ldots, \bar{n}, \overline{n+1}$. The vertices of $\Gamma_{n}^{B}$ are the $B$-diagonals, which are one of the two following kinds: diagonals joining antipodal pairs of points, and antipodal pairs of noncrossing diagonals. The diagonals joining antipodal points are all pairs of the form $\{i, \bar{i}\}$ satisfying $1 \leq i \leq n+1$. Simion calls such a $B$-diagonal a diameter. The $B$-diagonals that are antipodal pairs of noncrossing diagonals are either of the form $\{\{i, j\},\{\bar{i}, \bar{j}\}\}$ satisfying $1 \leq i<i+1<j \leq n+1$ or of the form $\{\{i, \bar{j}\},\{\bar{i}, j\}\}$ satisfying $1 \leq j<i \leq n+1$.

The simplicial complex $\Gamma_{n}^{B}$ is the family of sets of pairwise noncrossing $B$-diagonals. Simion showed the simplicial complex $\Gamma_{n}^{B}$ is the boundary complex of an $n$-dimensional convex polytope. This polytope is also known as the Bott-Taubes polytope [3] and the cyclohedron [16]. Simion also computed the face numbers and $h$-vector. See Theorem 1, Proposition 1 and Corollary 1 in [20], respectively. These numbers turn out to be identical with the face numbers and $h$-vector of any pulling triangulation of the Legendre polytope. We will discuss this polytope in the next subsection. We end with a fact that is implicit in the work of Simion [20, Section 3.3].

Lemma 2.1 (Simion). Each facet $\Gamma_{n}^{B}$ of Simion's type $B$ associahedron contains exactly one Bdiagonal of the form $\{i, \bar{i}\}$ connecting an antipodal pair of points. 
2.2. The Legendre polytope or "full" type $A$ root polytope. Consider an $(n+1)$-dimensional Euclidean space with orthonormal basis $\left\{e_{1}, e_{2}, \ldots, e_{n+1}\right\}$. The convex hull of the vertices $\pm 2 e_{1}$, $\ldots, \pm 2 e_{n+1}$ is an $(n+1)$-dimensional cross-polytope. The intersection of this cross-polytope with the hyperplane $x_{1}+x_{2}+\cdots+x_{n+1}=0$ is an $n$-dimensional centrally symmetric polytope $P_{n}$ first studied by Cho [7]. It is called the Legendre polytope in the work of Hetyei [13, since the polynomial $\sum_{j=0}^{n} f_{j-1} \cdot((x-1) / 2)^{j}$ is the $n$th Legendre polynomial, where $f_{i}$ is the number of $i$-dimensional faces in any pulling triangulation of the boundary of $P_{n}$. See Lemma 2.7 below. Furthermore, it is called the "full" type $A$ root polytope in the work of Ardila-Beck-Hoşten-Pfeifle-Seashore [1. It has $n(n+1)$ vertices consisting of all points of the form $e_{i}-e_{j}$ where $i \neq j$.

We use the shorthand notation $(i, j)$ for the vertex $e_{j}-e_{i}$ of the Legendre polytope $P_{n}$. We may think of these vertices as the set of all directed nonloop edges on the vertex set $\{1,2, \ldots, n+1\}$. A subset of these edges is contained in some face of $P_{n}$ exactly when there is no $i \in\{1,2, \ldots, n+1\}$ that is both the head and the tail of a directed edge. Equivalently, the faces are described as follows.

Lemma 2.2. The faces of the Legendre polytope $P_{n}$ are of the form $\operatorname{conv}(I \times J)=\operatorname{conv}(\{(i, j)$ : $i \in I, j \in J\})$ where $I$ and $J$ are two non-empty disjoint subsets of the set $\{1,2, \ldots, n+1\}$. The dimension of a face is given by $|I|+|J|-2$. A face is a facet if and only if the union of $I$ and $J$ is the set $\{1,2, \ldots, n+1\}$.

Especially, when the two sets $I$ and $J$ both have cardinality two, the associated face is geometrically a square. Furthermore, the other two-dimensional faces are equilateral triangles.

Affine independent subsets of vertices of faces of the Legendre polytope are easy to describe. A set $S=\left\{\left(i_{1}, j_{1}\right),\left(i_{2}, j_{2}\right), \ldots,\left(i_{k}, j_{k}\right)\right\}$ is a $(k-1)$-dimensional simplex if and only if, disregarding the orientation of the directed edges, the set $S$ contains no cycle, that is, it is a forest [13, Lemma 2.4].

The Legendre polytope $P_{n}$ contains the polytope $P_{n}^{+}$, defined as the convex hull of the origin and the set of points $e_{i}-e_{j}$, where $i<j$. The polytope $P_{n}^{+}$was first studied by Gelfand, Graev and Postnikov [10] and later by Postnikov [19]. Some of the results on $P_{n}^{+}$may be easily generalized to $P_{n}$.

2.3. Pulling triangulations. The notion of pulling triangulations is originally due to Hudson [14, Lemma 1.4]. For more modern formulations, see [21, Lemma 1.1] and [2, End of Section 2]. We refer to [13, Section 2.3] for the version presented here.

For a polytopal complex $\mathcal{P}$ and a vertex $v$ of $\mathcal{P}$, let $\mathcal{P}-v$ be the complex consisting of all faces of $\mathcal{P}$ not containing the vertex $v$. Also for a facet $F$ let $\mathcal{P}(F)$ be the complex of all faces of $\mathcal{P}$ contained in $F$.

Definition 2.3 (Hudson). Let $\mathcal{P}$ be a polytopal complex and let $<$ be a linear order on the set $V$ of its vertices. The pulling triangulation $\triangle(\mathcal{P})$ with respect to $<$ is defined recursively as follows. We set $\triangle(\mathcal{P})=\mathcal{P}$ if $\mathcal{P}$ consists of a single vertex. Otherwise let $v$ be the least element of $V$ with respect to $<$ and set

$$
\triangle(\mathcal{P})=\triangle(\mathcal{P}-v) \cup \bigcup_{F}\{\operatorname{conv}(\{v\} \cup G): G \in \triangle(\mathcal{P}(F))\}
$$


where the union runs over the facets $F$ not containing $v$ of the maximal faces of $\mathcal{P}$ which contain $v$. The triangulations $\triangle(\mathcal{P}-v)$ and $\triangle(\mathcal{P}(F))$ are with respect to the order $<$ restricted to their respective vertex sets.

Theorem 2.4 (Hudson). The pulling triangulation $\triangle(\mathcal{P})$ is a triangulation of the polytopal complex $\mathcal{P}$ without introducing any new vertices.

In particular, any pulling triangulation of the boundary of $P_{n}$ is compressed as defined by Stanley [21, and has the same face numbers [13, Corollary 4.11]. This important fact and the analogous statement for $P_{n}^{+}$is a direct consequence of the following two fundamental results [11, 12, 21.

Proposition 2.5 (Stanley). Suppose that one of the vertices of a polytope $P$ is the origin and that the matrix whose rows are the coordinates of the vertices of $P$ is totally unimodular. Let $<$ be any ordering on the vertex set of $P$ such that the origin is the least vertex with respect to $<$. Then the pulling order $<$ is compressed, that is, all of the facets in the induced triangulation have the same relative volume.

Theorem 2.6 (Heller). The incidence matrix of a directed graph is totally unimodular.

2.4. Face vectors of pulling triangulations of the Legendre polytope. Among all triangulations of the boundary of the Legendre polytope $P_{n}$ obtained by pulling the vertices, counting faces is most easily performed for the lexicographic triangulation in which we pull $(i, j)$ before $\left(i^{\prime}, j^{\prime}\right)$ exactly when $i<i^{\prime}$ or when $i=i^{\prime}$ and $j<j^{\prime}$. Counting faces in this triangulation amounts to counting lattice paths; see [13, Lemma 5.1] and [1, Proposition 17]. From this we obtain the following expression for the face numbers [13, Theorem 5.2].

Lemma 2.7 (Hetyei). For any pulling triangulation of the boundary of $P_{n}$, the number $f_{j-1}$ of $(j-1)$ dimensional faces is

$$
f_{j-1}=\left(\begin{array}{c}
n+j \\
j
\end{array}\right)\left(\begin{array}{l}
n \\
j
\end{array}\right) \quad \text { for } 0 \leq j \leq n
$$

It was first noted in [9] that these face numbers are the same as that of Simion's type $B$ associahedron $\Gamma_{n}^{B}$. Theorem 6.1 will explicitly explain this fact by showing that $\Gamma_{n}^{B}$ can be realized as a pulling triangulation of the Legendre polytope.

\section{The FlaG PROPERTY}

Recall that a simplicial complex is a flag complex if every minimal nonface has two elements. The main result of this section is the following.

Theorem 3.1. Every pulling triangulation of the boundary of the Legendre polytope $P_{n}$ is a flag simplicial complex.

To prove this theorem and Theorem 6.1, we need the following observation. 
Lemma 3.2. Let $\left\{x_{1}, x_{2}, y_{1}, y_{2}\right\}$ be a four element subset of the set $\{1,2, \ldots, n+1\}$. Then the set $\left\{x_{1}, x_{2}\right\} \times\left\{y_{1}, y_{2}\right\}=\left\{\left(x_{1}, y_{1}\right),\left(x_{1}, y_{2}\right),\left(x_{2}, y_{2}\right),\left(x_{2}, y_{1}\right)\right\}$ is the vertex set of a square face of the Legendre polytope $P_{n}$, and the sets $\left\{\left(x_{1}, y_{1}\right),\left(x_{2}, y_{2}\right)\right\}$ and $\left\{\left(x_{1}, y_{2}\right),\left(x_{2}, y_{1}\right)\right\}$ are the diagonals of this square. For any pulling triangulation the diagonal containing the vertex that was pulled first is an edge of the triangulation and the other diagonal is not an edge.

Theorem 3.1 may be rephrased as follows.

Theorem 3.3. Let $<$ be any order on the vertices of the Legendre polytope $P_{n}$ and consider the pulling triangulation of the boundary of $P_{n}$ induced by this order. Suppose we are given a set of vertices $\left\{\left(u_{1}, v_{1}\right),\left(u_{2}, v_{2}\right), \ldots,\left(u_{k}, v_{k}\right)\right\}$ such that any pair $\left\{\left(u_{i}, v_{i}\right),\left(u_{j}, v_{j}\right)\right\}$ is an edge in the pulling triangulation. Then this set is a face of the pulling triangulation.

Proof. Assume that the pulling order on the set of vertices is given by $\left(u_{1}, v_{1}\right)<\left(u_{2}, v_{2}\right)<\cdots<$ $\left(u_{k}, v_{k}\right)$. We prove the statement by induction on $k$. The statement is directly true for $k \leq 2$. Assume from now on $k \geq 3$. Observe first that the sets $\left\{u_{1}, \ldots, u_{k}\right\}$ and $\left\{v_{1}, \ldots, v_{k}\right\}$ must be disjoint. Indeed, if for any $i \neq j$ we have $u_{i}=v_{j}$ then the pair of vertices $\left\{\left(u_{i}, v_{i}\right),\left(u_{j}, v_{j}\right)\right\}$ is not an edge, contradicting our assumption. Thus our set of vertices is contained in the face $\operatorname{conv}\left(\left\{u_{1}, \ldots, u_{k}\right\} \times\left\{v_{1}, \ldots, v_{k}\right\}\right)$ of the polytope $P_{n}$. Note that the lists $u_{1}, \ldots, u_{k}$ and $v_{1}, \ldots, v_{k}$ may contain repeated elements.

We next show that $\left(u_{1}, v_{1}\right)$ is the least element with respect of the order $<$ in the set $\left\{u_{1}, \ldots, u_{k}\right\} \times$ $\left\{v_{1}, \ldots, v_{k}\right\}$. To prove this, suppose $\left(u_{i}, v_{j}\right)$ is the least element. If $i=j$ then either $i=1$ or we may use that we were given $\left(u_{1}, v_{1}\right)<\left(u_{i}, v_{i}\right)$ for all $i>1$. If $i \neq j$ but $u_{i}=u_{j}$ then we have $\left(u_{i}, v_{j}\right)=\left(u_{j}, v_{j}\right)$ and again we are done since $\left(u_{j}, v_{j}\right)>\left(u_{1}, v_{1}\right)$ if $j>1$. Similarly, if $i \neq j$ but $v_{i}=v_{j}$ then we may use $\left(u_{i}, v_{j}\right)=\left(u_{i}, v_{i}\right)$. Finally if $i \neq j, u_{i} \neq u_{j}$ and $v_{i} \neq v_{j}$ hold, then apply Lemma 3.2 to the square with vertex set $\left(u_{i}, v_{i}\right),\left(u_{i}, v_{j}\right),\left(u_{j}, v_{j}\right)$ and $\left(u_{j}, v_{i}\right)$. Since $\left(u_{i}, v_{j}\right)$ is the least with respect to the pulling order, the diagonal $\left\{\left(u_{i}, v_{i}\right),\left(u_{j}, v_{j}\right)\right\}$ is not an edge of the triangulation, contradicting our assumption. Hence we conclude $\left(u_{1}, v_{1}\right)$ is the least vertex in the set $\left\{u_{1}, \ldots, u_{k}\right\} \times\left\{v_{1}, \ldots, v_{k}\right\}$.

We claim the smallest face of the boundary of $P_{n}$ containing the vertex set $\left\{\left(u_{2}, v_{2}\right), \ldots,\left(u_{k}, v_{k}\right)\right\}$ does not contain the vertex $\left(u_{1}, v_{1}\right)$. Assume, by way of contradiction, that $\left\{u_{1}, u_{2}, \ldots, u_{k}\right\}=$ $\left\{u_{2}, \ldots, u_{k}\right\}$ and $\left\{v_{1}, v_{2}, \ldots, v_{k}\right\}=\left\{v_{2}, \ldots, v_{k}\right\}$ hold. Then there is an index $i>1$ and an index $j>1$ such that $u_{1}=u_{i}$ and $v_{1}=v_{j}$ hold. Note that we must also have $v_{1} \neq v_{i}$ and $u_{1} \neq u_{j}$. Consider the square face with the following four vertices: $\left(u_{1}, v_{1}\right),\left(u_{1}, v_{i}\right)=\left(u_{i}, v_{i}\right),\left(u_{j}, v_{i}\right)$ and $\left(u_{j}, v_{j}\right)=\left(u_{j}, v_{1}\right)$. The first vertex that was pulled is $\left(u_{1}, v_{1}\right)$. By Lemma 3.2 the edge $\left\{\left(u_{i}, v_{i}\right),\left(u_{j}, v_{j}\right)\right\}$ is not an edge in the pulling triangulation, contradicting our assumptions.

By the induction hypothesis the set $\left\{\left(u_{2}, v_{2}\right), \ldots,\left(u_{k}, v_{k}\right)\right\}$ is a face in the polytopal complex $\partial P_{n}-$ $\left\{w: w \leq\left(u_{1}, v_{1}\right)\right\}$. By definition, we obtain that $\left\{\left(u_{1}, v_{1}\right)\right\} \cup\left\{\left(u_{2}, v_{2}\right), \ldots,\left(u_{k}, v_{k}\right)\right\}$ is a face of the pulling triangulation.

Since the Cartesian product of an $m$-dimensional simplex and an $n$-dimensional simplex is a face of the Legendre polytope of dimension $m+n+1$, we obtain the following corollary.

Corollary 3.4. Every pulling triangulation of the Cartesian product of two simplices is a flag complex. 

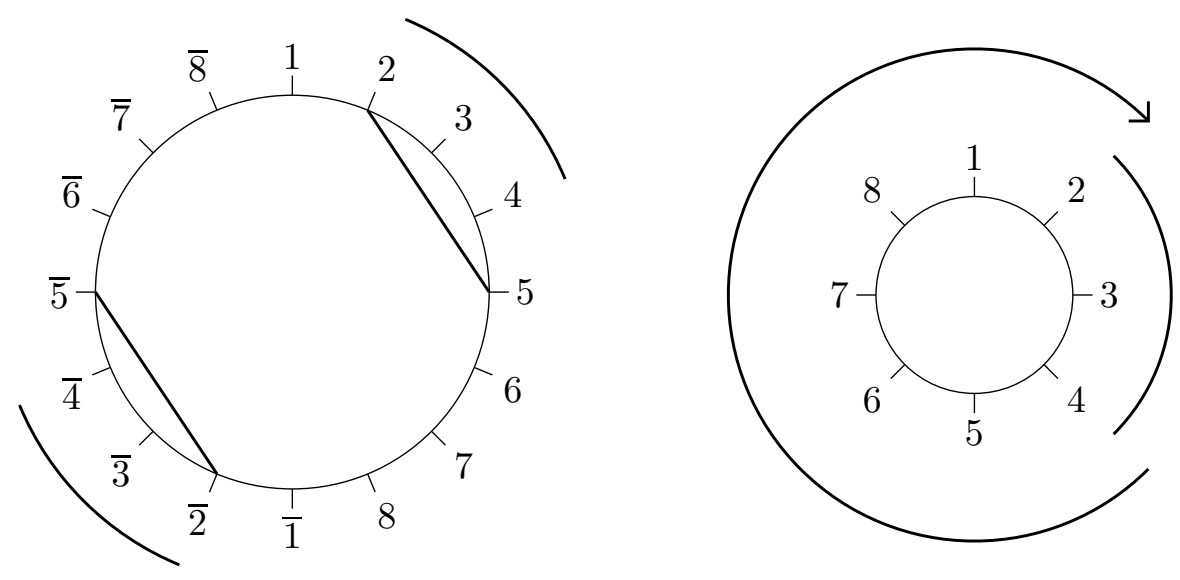

Figure 1. The arc representation of the $B$-diagonal consisting of the two diagonals $\{2,5\}$ and $\{\overline{2}, \overline{5}\}=\{10,13\}$ is the two arcs $[2,4]$ and $[\overline{2}, \overline{4}]=[10,12]$. By considering the arcs modulo $n+1=8$ (see the second circle) we obtain that this $B$-diagonal is represented by the arrow $(4,2)$.

\section{The arc Representation of $\Gamma_{n}^{B}$}

In this section we describe a representation of $\Gamma_{n}^{B}$ as a simplicial complex whose vertices are centrally symmetric pairs of "arcs" on a circle. This representation has a natural circular symmetry.

Consider a regular $(2 n+2)$-gon whose vertices are labeled $1,2, \ldots, n+1, \overline{1}, \overline{2}, \ldots, \overline{n+1}$ in the clockwise order. Identify each vertex $\bar{i}$ with $n+1+i$ for $i=1,2, \ldots, n+1$. Subject to this identification, each $B$-diagonal, that is, a pair of diagonals, may be represented as an unordered pair of diagonals of the form $\{\{u, v\},\{u+n+1, v+n+1\}\}$ for some $\{u, v\} \subseteq\{1,2, \ldots, 2 n+2\}$, where addition is modulo $2 n+2$. For $B$-diagonals $\{k, \bar{k}\}$ joining antipodal points, the unordered pair $\{\{k, k+n+1\},\{k+$ $n+1, k+2 n+2\}\}$ contains two copies of the same two-element set.

For any two points $x$ and $y$ on the circle $\mathbb{R} /(2 n+2) \mathbb{Z}$ which are not antipodal, let $[x, y]$ denote the shortest arc from $x$ to $y$.

Definition 4.1. We define the arc-representation on the vertices of $\Gamma_{n}^{B}$ as follows. Subject to the above identifications, represent the B-diagonal $\{\{u, v\},\{u+n+1, v+n+1\}\}$ with the centrally symmetric pair of arcs $\{[u, v-1],[u+n+1, v+n]\}$ on the circle $\mathbb{R} /(2 n+2) \mathbb{Z}$.

The representation above is well-defined: for any pair of vertices $u$ and $v$, the arc $[u, v-1]$ and the arc $[u+n+1, v+n]$ form a centrally symmetric pair, so the definition is independent of the selection of the element of $\{\{u, v\},\{u+n+1, v+n+1\}\}$. Note that for $B$-diagonals of the form $\{\{k, n+1+k\},\{k, n+1+k\}\}$ corresponding to antipodal pairs of points, the union of the arcs $[k, k+n]$ and $[k+n+1, k-1]$ is not the full circle.

See Figure 1 for an example where $n=7$ with the $B$-diagonal $\{\{2,5\},\{\overline{2}, \overline{5}\}\}$. 
Lemma 4.2. The arc-representation of the vertices of $\Gamma_{n}^{B}$ is one-to-one: distinct B-diagonals are mapped to distinct centrally symmetric pairs of arcs.

Indeed, the pair $\{[u, v-1],[u+n+1, v+n]\}$ can only be the image of the unordered pair $\{\{u, v\},\{u+$ $n+1, v+n+1\}\}$.

The following theorem plays an important role in connecting the type $B$ associahedron with the Legendre polytope.

Theorem 4.3. The $B$-diagonal represented by the pair of arcs $\left\{\left[u_{1}, v_{1}-1\right],\left[u_{1}+n+1, v_{1}+n\right]\right\}$ and the $B$-diagonal represented by the pair of arcs $\left\{\left[u_{2}, v_{2}-1\right],\left[u_{2}+n+1, v_{2}+n\right]\right\}$ are noncrossing if and only if for either arc $I \in\left\{\left[u_{1}, v_{1}-1\right],\left[u_{1}+n+1, v_{1}+n\right]\right\}$ and for either arc $J \in\left\{\left[u_{2}, v_{2}-1\right],\left[u_{2}+n+1, v_{2}+n\right]\right\}$, the arcs $I$ and $J$ are either nested or disjoint.

Proof. Assume first that the two $B$-diagonals cross. Since we may replace $\left\{u_{i}, v_{i}\right\}$ with $\left\{u_{i}+n+1, v_{i}+\right.$ $n+1\}$ if necessary, without loss of generality we may assume that the diagonal $\left\{u_{1}, v_{1}\right\}$ crosses the diagonal $\left\{u_{2}, v_{2}\right\}$. Exactly one of the endpoints of the diagonal $\left\{u_{2}, v_{2}\right\}$ must then belong to the arc $\left[u_{1}+1, v_{1}-1\right]$ and the other one does not belong even to the larger arc $\left[u_{1}, v_{1}\right]$. If $u_{2} \in\left[u_{1}+1, v_{1}-1\right]$ and $v_{2} \notin\left[u_{1}, v_{1}\right]$ then we have

$$
\left[u_{1}, v_{1}-1\right] \cap\left[u_{2}, v_{2}-1\right]=\left[u_{2}, v_{1}-1\right] .
$$

This arc contains $u_{2}$, but does not contain $u_{1}$ (since $u_{2} \in\left[u_{1}+1, v_{1}-1\right]$ ), nor does it contain $v_{2}-1 \notin\left[u_{1}, v_{1}-1\right]$. The $\operatorname{arcs}\left[u_{1}, v_{1}-1\right]$ and $\left[u_{2}, v_{2}-1\right]$ do not contain each other and they are not disjoint.

If $v_{2} \in\left[u_{1}+1, v_{1}-1\right]$ and $u_{2} \notin\left[u_{1}, v_{1}\right]$ then we have

$$
\left[u_{1}, v_{1}-1\right] \cap\left[u_{2}, v_{2}-1\right]=\left[u_{1}, v_{2}-1\right] .
$$

Similar to the previous case, we obtain that the arcs $\left[u_{1}, v_{1}-1\right]$ and $\left[u_{2}, v_{2}-1\right]$ do not contain each other and they are not disjoint.

Assume next that the two diagonals do not cross. If $u_{2} \in\left[u_{1}+1, v_{1}-1\right]$ then we must have $v_{2} \in\left[u_{2}+1, v_{1}\right]$, implying

$$
\left[u_{1}, v_{1}-1\right] \cap\left[u_{2}, v_{2}-1\right]=\left[u_{2}, v_{2}-1\right] .
$$

Similarly, if $v_{2} \in\left[u_{1}+1, v_{1}-1\right]$ then we must have $u_{2} \in\left[u_{1}, v_{2}\right]$, implying

$$
\left[u_{1}, v_{1}-1\right] \cap\left[u_{2}, v_{2}-1\right]=\left[u_{2}, v_{2}-1\right] .
$$

Finally, if neither $u_{2}$ nor $v_{2}$ belongs to $\left[u_{1}+1, v_{1}-1\right]$ then either the arc $\left[u_{2}, v_{2}-1\right]$ contains the arc $\left[u_{1}, v_{1}-1\right]$ or it is disjoint from it. The above argument remains valid if we replace either (or both) of $\left[u_{1}, v_{1}-1\right]$ and $\left[u_{2}, v_{2}-1\right]$ with the $\operatorname{arc}\left[u_{1}+n+1, v_{1}+n\right]$ or $\left[u_{2}+n+1, v_{2}+n\right]$, respectively.

Corollary 4.4. The $B$-diagonal represented by the pair of arcs $\left\{\left[u_{1}, v_{1}-1\right],\left[u_{1}+n+1, v_{1}+n\right]\right\}$ and the $B$-diagonal represented by the pair of arcs $\left\{\left[u_{2}, v_{2}-1\right],\left[u_{2}+n+1, v_{2}+n\right]\right\}$ are noncrossing if and only the set $\left[u_{1}, v_{1}-1\right] \cup\left[u_{1}+n+1, v_{1}+n\right]$ and the set $\left[u_{2}, v_{2}-1\right] \cup\left[u_{2}+n+1, v_{2}+n\right]$ are nested or disjoint. 


\section{Embedding $\Gamma_{n}^{B}$ AS A FAMily of Simplices ON $\partial P_{n}$}

In this section we describe a way to represent the boundary complex $\Gamma_{n}^{B}$ of Simion's type $B$ associahedron as a family of simplices on the vertex set of the Legendre polytope $P_{n}$. We do this so that each simplex is contained in a face of the boundary $\partial P_{n}$ of $P_{n}$. In Section 6 we will prove that our map represents the boundary complex of the type $B$ associahedron as a pulling triangulation of $\partial P_{n}$.

We begin by defining a bijection between the vertex set of $\Gamma_{n}^{B}$ and that of $P_{n}$. Recall that we use the shorthand notation $(i, j)$ for the vertex $e_{j}-e_{i}$ of $P_{n}$. We refer to $(i, j)$ as the arrow from $i$ to $j$. Using the term "arrow" as opposed to "directed edge" will eliminate the confusion that $e_{j}-e_{i}$ is a vertex of $P_{n}$. Instead we think of it as an arrow from $i$ to $j$ in the complete directed graph on the vertex set $\{1,2, \ldots, n+1\}$ having no loops.

Definition 5.1. Let $\{[i, j],[\bar{i}, \bar{j}]\}$ be the arc representation of a $B$-diagonal in $\Gamma_{n}^{B}$, where $1 \leq i \leq n+1$ and $i<j$. Define the arrow representation of this $B$-diagonal in $P_{n}$ to be the arrow $(j, i)$.

In other words, the arrow encodes the complement of the image of the arcs in the circle $\mathbb{R} /(n+1) \mathbb{Z}$. We refer to the second circle in Figure 1 for the continuation of the example of the $B$-diagonal $\{\{2,5\},\{\overline{2}, \overline{5}\}\}$.

When making this definition explicit for a $B$-diagonal $\{\{i, j\},\{\bar{i}, \bar{j}\}\}$, we obtain several cases:

(1) For each $i$ satisfying $2 \leq i \leq n+1$, represent the $B$-diagonal $\{i, \bar{i}\}$ connecting two antipodal points with the arrow $(i-1, i)$. Represent the $B$-diagonal $\{1, \overline{1}\}$ with the arrow $(n+1,1)$.

(2) For each $i$ and $j$ satisfying $1 \leq i<i+1<j \leq n+1$, represent the $B$-diagonal $\{\{i, j\},\{\bar{i}, \bar{j}\}\}$ with the arrow $(j-1, i)$.

(3) For each $i$ and $j$ satisfying $2 \leq j<i \leq n+1$, represent the $B$-diagonal $\{\{i, \bar{j}\},\{\bar{i}, j\}\}$ with the arrow $(j-1, i)$. For each $i$ satisfying $2 \leq i \leq n+1$, represent the $B$-diagonal $\{\{1, \bar{i}\},\{\overline{1}, i\}\}$ with the arrow $(n+1, i)$.

This representation yields a bijection between $B$-diagonals and arrows. The inverse map is given as follows:

(a) For $i$ satisfying $1 \leq i \leq n$, the arrow $(i, i+1)$ represents the $B$-diagonal $\{i+1, \overline{i+1}\}$ and the arrow $(n+1,1)$ represents the $B$-diagonal $\{1, \overline{1}\}$.

(b) For each $i$ and $j$ satisfying $1 \leq i<j \leq n$, the arrow $(j, i)$ represents the $B$-diagonal $\{\{i, j+$ $1\},\{\bar{i}, \overline{j+1}\}\}$.

(c) For each $i$ and $j$ satisfying $1 \leq j<i-1<i \leq n+1$, the arrow $(j, i)$ represents the $B$-diagonal $\{\{i, \overline{j+1}\},\{\bar{i}, j+1\}\}$, and for $2 \leq i \leq n+1$, the arrow $(n+1, i)$ represents the $B$-diagonal $\{\{1, \bar{i}\},\{\overline{1}, i\}\}$.

The $B$-diagonals in item (1) of Definition 5.1 may be thought of as a "degenerate case" of the $B$-diagonals in item (3). In the case when $i=j$, the set $\{\{\bar{i}, j\},\{i, \bar{j}\}\}$ becomes a singleton, and the rules in item (1) may be obtained by extending the rules in item (3) in an obvious way. On the other 
hand, Definition 5.1 has a simpler form in terms of the arc-representation described in Section 4 and in terms of the following continuous map.

Definition 5.2. Define the map $\pi: \mathbb{R} /(2 n+2) \mathbb{Z} \longrightarrow \mathbb{R} /(n+1) \mathbb{Z}$ to be the modulo $n+1$ map. Furthermore, identify the circle $\mathbb{R} /(n+1) \mathbb{Z}$ with the half-open interval $(0, n+1]$. Thus $\pi$ sends each $x \in(0, n+1]$ to $x$ and each $x \in(n+1,2 n+2]$ to $x-n-1$.

Observe that the map $\pi$ depends on $n$. However, we suppress this dependency by not writing $\pi_{n}$. Also observe that the map $\pi$ is a two-to-one mapping: for each $y \in \mathbb{R} /(n+1) \mathbb{Z}$ we have $\left|\pi^{-1}(y)\right|=2$.

Remark 5.3. For any pair of $\operatorname{arcs}\{[u, v-1],[u+n+1, v+n]\}$ there is a unique way to select $u$ to be an element of the set $\{1,2, \ldots, n+1\}$, that is, $\pi(u)=u$. We may distinguish two cases depending upon whether the arc $[u, v-1]$ is a subset of the arc $[u, n+1]$ or not.

(i) If $[u, v-1] \subseteq[u, n+1]$ then visualize the set $\pi([u, v-1])=\pi([u+n+1, v+n])$ as the subinterval $[u, v-1]$ of $(0, n+1]$. The direction of both arcs $[u, v-1]$ and $[u+n+1, v+n]$ corresponds to parsing the interval $[u, v-1]$ in increasing order. Adding the associated backward arrow $(v-1, u)$ closes a directed cycle with this directed interval.

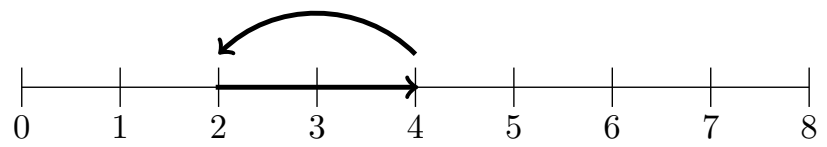

As an example, when $n=7$ then the $B$-diagonal $\{\{2,5\},\{2, \overline{5}\}\}$ is represented by the backward arrow $(4,2)$ as drawn above on the interval $(0,8]$.

(ii) If the arc $[u, v-1]$ is not contained in the arc $[u, n+1]$ then $n+1<v-1<u+n+1$. The integer $\pi(v-1)=v-1-(n+1)$ is congruent to $v-1$ modulo $(n+1)$ and satisfies $1 \leq \pi(v-1)<u$. The image of the arc $[u, v-1]$ under $\pi$, that is, $\pi([u, v-1])=\pi([u+n+1, v+n])$ is then the subset $(0, \pi(v)-1] \cup[u, n+1]$ of the interval $(0, n+1]$. We may consider $(0, \pi(v)-1] \cup[u, n+1]$ as a "wraparound interval" modulo $n+1$ from $u$ to $\pi(v-1)$. The direction of both pieces corresponds to listing the elements of this "wraparound interval" in increasing order modulo $n+1$. Adding the associated forward arrow $(\pi(v-1), u)$ closes a directed cycle with the directed wraparound interval.

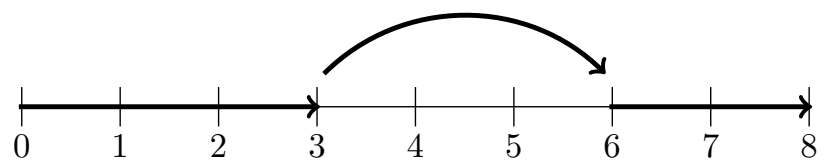

For instance, when $n=7$ the $B$-diagonal $\{\{4, \overline{6}\},\{\overline{4}, 6\}\}$ yields the forward arrow $(3,6)$.

Proposition 5.4. The B-diagonal represented by the arrow $\left(\pi\left(v_{1}-1\right), \pi\left(u_{1}\right)\right)$ and the B-diagonal represented by the arrow $\left(\pi\left(v_{2}-1\right), \pi\left(u_{2}\right)\right)$ are noncrossing if and only if the images $\pi\left(\left[u_{1}, v_{1}-1\right]\right)$ and $\pi\left(\left[u_{2}, v_{2}-1\right]\right)$ are disjoint or contain each other.

Proof. The statement is an easy consequence of the following observation. Both arcs of the arc representation $\{[u, v-1],[u+n+1, v+n]\}$ of a $B$-diagonal are mapped onto the same $\operatorname{arc}[\pi(u), \pi(v-1)]$ by $\pi$ and $[u, v-1] \cup[u+n+1, v+n]=\pi^{-1}([\pi(u), \pi(v-1)])$. 
Next we translate the noncrossing conditions for $B$-diagonals into conditions for the arrows representing them.

Proposition 5.5. Suppose a pair of B-diagonals is represented by a pair of arrows as defined in Definition 5.1. These B-diagonals cross if and only if one of the following conditions is satisfied:

(1) Both arrows are backward and they cross.

(2) Both arrows are forward and they do not nest.

(3) One arrow is forward, the other one is backward, and the backward arrow nests or crosses the forward arrow.

(4) The head of one arrow is the tail of the other arrow.

Proof. Suppose the arc representations of the two $B$-diagonals are $\left\{\left[u_{1}, v_{1}-1\right],\left[u_{1}+n+1, v_{1}+n\right]\right\}$ and $\left\{\left[u_{2}, v_{2}-1\right],\left[u_{2}+n+1, v_{2}+n\right]\right\}$, respectively. By Proposition [5.4, the represented $B$-diagonals are crossing if and only if the images $\pi\left(\left[u_{1}, v_{1}-1\right]\right)$ and $\pi\left(\left[u_{2}, v_{2}-1\right]\right)$ are not disjoint and do not contain each other.

We will consider three cases, depending on the direction of the two arrows $\left(\pi\left(v_{1}-1\right), \pi\left(u_{1}\right)\right)$ and $\left(\pi\left(v_{2}-1\right), \pi\left(u_{2}\right)\right)$. These arrows are either both forward, both backward or have opposite directions.

If both arrows are backward then neither the image $\pi\left(\left[u_{1}, v_{1}-1\right]\right)$ nor the image $\pi\left(\left[u_{2}, v_{2}-1\right]\right)$ contain the point $n+1$. Two such intervals intersect nontrivially in an interval of positive length exactly when the corresponding arrows cross. They intersect in a single point exactly when there is a vertex that is the tail of one of the arrows and the head of the other arrow.

If both arrows are forward then both images $\pi\left(\left[u_{1}, v_{1}-1\right]\right)$ and $\pi\left(\left[u_{2}, v_{2}-1\right]\right)$ contain the point $n+1$, so they cannot be disjoint. They do not contain each other exactly when the corresponding arrows are not nested. Note that a pair of forward arrows such that the head of one arrow is the same as the tail of the other is particular example of a pair of nonnested arrows.

Assume finally that one of the arrows, say $\left(\pi\left(v_{1}-1\right), \pi\left(u_{1}\right)\right)$, is a backward arrow and the other one, say $\left(\pi\left(v_{2}-1\right), \pi\left(u_{2}\right)\right)$, is a forward arrow. The image $\pi\left(\left[u_{2}, v_{2}-1\right]\right)$ cannot be a subset of $\pi\left(\left[u_{1}, v_{1}-1\right]\right)$ as the first image contains the point $n+1$ whereas the second does not. The image $\pi\left(\left[u_{1}, v_{1}-1\right]\right)$ is the interval $\left[\pi\left(u_{1}\right), \pi\left(v_{1}-1\right)\right]$, whereas the image $\pi\left(\left[u_{2}, v_{2}-1\right]\right)$ is the union $\left(0, \pi\left(v_{2}-1\right)\right] \cup\left[\pi\left(u_{2}\right), n+1\right]$. The intersection of these two sets has either zero, one or two connected components. If one component is a single point then this point is the head of one arrow and the tail of the other. If both components are non-trivial intervals then the backward arrow nests the forward arrow. If they intersect in one interval but the image $\pi\left(\left[u_{1}, v_{1}-1\right]\right)$ does not contain the image $\pi\left(\left[u_{2}, v_{2}-1\right]\right)$ then the two arrows cross. Finally, if the image $\pi\left(\left[u_{1}, v_{1}-1\right]\right)$ does contain the image $\pi\left(\left[u_{2}, v_{2}-1\right]\right)$ then the arrows neither cross nor nest.

An immediate consequence of Proposition 5.5 and Lemma 2.2 is the following corollary.

Corollary 5.6. Noncrossing sets of B-diagonals correspond to subsets of vertices contained in a facet of the Legendre polytope $P_{n}$. 


\begin{tabular}{|c|c|c|c|}
\hline Order of nodes & Arrow first pulled & Edge & Not an edge \\
\hline$x_{1}<x_{2}<y_{1}<y_{2}$ & $\left(x_{2}, y_{1}\right)$ & $x_{1} \widehat{x_{2} y_{1}} y_{2}$ & $x_{1} x_{2} y_{1} y_{2}$ \\
\hline$x_{1}<y_{1}<x_{2}<y_{2}$ & $\left(x_{2}, y_{1}\right)$ & $x _ { 1 } \longdiv { y _ { 1 } x _ { 2 } } y _ { 2 }$ & $\vec{x}_{1} \vec{y}_{1} x_{2} \vec{y}_{2}$ \\
\hline$x_{1}<y_{1}<y_{2}<x_{2}$ & $\left(x_{2}, y_{2}\right)$ & 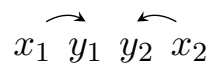 & $x _ { 1 } \longdiv { y _ { 1 } y _ { 2 } } x _ { 2 }$ \\
\hline$y_{1}<x_{1}<x_{2}<y_{2}$ & $\left(x_{1}, y_{1}\right)$ & $\widehat{y_{1} x_{1}} x_{2} \vec{y}_{2}$ & $\overbrace{y_{1} x_{1} x_{2}} y_{2}$ \\
\hline$y_{1}<x_{1}<y_{2}<x_{2}$ & $\left(x_{1}, y_{1}\right)$ or $\left(x_{2}, y_{2}\right)$ & $\widetilde{y_{1} x_{1}} \widetilde{y_{2}} x_{2}$ & $\widehat{y_{1} \widehat{x_{1}} \widehat{y_{2}}} x_{2}$ \\
\hline$y_{1}<y_{2}<x_{1}<x_{2}$ & $\left(x_{1}, y_{2}\right)$ & 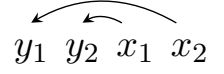 & $\stackrel{\gtrless}{y_{1} y_{2} x_{1}} x_{2}$ \\
\hline
\end{tabular}

TABLE 1. Pairs of arrows that are edges or minimal nonfaces

\section{The type $B$ ASSOCiahedron REPRESENTED AS A PUlling tRIANGUlation}

In this section we show that the arc representation given in Definition 5.1 constitutes Simion's type $B$ associahedron $\Gamma_{n}^{B}$ as a pulling triangulation of the boundary of the Legendre polytope $P_{n}$. Our main result is the following.

Theorem 6.1. Let $<$ be any linear order on the vertex set of $P_{n}$ subject to the following conditions:

(1) $\left(x_{1}, y_{1}\right)<\left(x_{2}, y_{2}\right)$ whenever $x_{1}-y_{1}>0>x_{2}-y_{2}$.

(2) On the subset of vertices $(x, y)$ satisfying $x<y$, we have $\left(x_{1}, y_{1}\right)<\left(x_{2}, y_{2}\right)$ whenever the interval $\left[x_{1}, y_{1}\right]=\left\{x_{1}, x_{1}+1, \ldots, y_{1}\right\}$ is contained in the interval $\left[x_{2}, y_{2}\right]$.

(3) On the subset of vertices $(x, y)$ satisfying $x>y$, we have $\left(x_{1}, y_{1}\right)<\left(x_{2}, y_{2}\right)$ whenever the interval $\left[y_{1}, x_{1}\right]$ is contained in the interval $\left[y_{2}, x_{2}\right]$.

Then the arc representation of $\Gamma_{n}^{B}$ given in Definition [5.1 is a pulling triangulation of the boundary of the Legendre polytope $P_{n}$ with respect to $<$.

Proof. Fix any pulling order < satisfying the above conditions. The first condition requires all backward arrows to precede all forward arrows. The next two conditions require that for a pair of nested arrows of the same direction the nested arrow should precede the nesting arrow.

Recall that $\Gamma_{n}^{B}$ is a flag complex and its minimal nonfaces are the pairs of crossing $B$-diagonals. By Theorem 3.1 the pulling triangulation we defined is also a flag complex. It suffices to show that the 
minimal nonfaces are in bijection. Equivalently, for any pair of arrows $\left\{\left(x_{1}, y_{1}\right),\left(x_{2}, y_{2}\right)\right\}$ that form an edge in the pulling triangulation of $P_{n}$, these arrows correspond to a pair of noncrossing $B$-diagonals in $\Gamma_{n}^{B}$. By Proposition 5.5 this amounts to showing the following: backward arrows cannot cross, forward arrows must nest, and for a pair of arrows of opposite direction the backward arrow cannot cross or nest the forward arrow.

Given a four-element subset $\left\{x_{1}, x_{2}, y_{1}, y_{2}\right\}$ of $\{1,2, \ldots, n+1\}$, where $x_{1}<x_{2}$ and $y_{1}<y_{2}$, consider the four arrows in the set $\left\{x_{1}, x_{2}\right\} \times\left\{y_{1}, y_{2}\right\}$. As seen in Lemma 3.2, these four arrows form the vertex set of a square face of the Legendre polytope $P_{n}$, and only the diagonal which contains the first vertex to be pulled is an edge of the pulling triangulation. Table 1 lists all six possible orderings of this four element set.

In each of the six cases we note which vertex is pulled first, which diagonal of the square becomes an edge in the triangulation and which diagonal does not become an edge. Note that in the fifth row of Table 1 we have two possibilities for selecting the vertex to be pulled first. However, these two vertices belong to the same diagonal. In every case we obtain that none of the pairs of arrows with distinct heads and tails that is an edge corresponds to a pair of crossing $B$-diagonals.

As a corollary we obtain Simion's polytopal result.

Corollary 6.2 (Simion). The Simion type B associahedron $\Gamma_{n}^{B}$ is the boundary complex of a simplicial polytope.

Since the associahedron of type $A$ is the link of a $B$-diagonal of the form $\{i, \bar{i}\}$, we obtain the following classical result; see the work of Haiman, Lee [15] and Stasheff. For a brief history, see the introduction of [5].

Corollary 6.3. The associahedron is the boundary complex of a simplicial polytope.

We end this section by describing the structure of all facets of the Simion's type $B$ associahedron in terms of arrows.

Theorem 6.4. A set of arrows $S=\left\{\left(x_{1}, y_{1}\right), \ldots,\left(x_{n}, y_{n}\right)\right\}$ represents a facet of Simion's type $B$ associahedron $\Gamma_{n}^{B}$ if and only if the following conditions are satisfied:

(1) There is exactly one $k$ satisfying $1 \leq k \leq n+1$ such that $(k-1, k)$ (or $(n+1,1)$ if $k=1)$ belongs the set $S$. We call this $k$ the type of the facet.

(2) Backward arrows do not nest any forward arrow, in particular, they cannot nest $(k-1, k)$ if $k>1$.

(3) If $k=1$ then there is no forward arrow in the set $S$.

(4) Forward arrows must nest. In particular, if $k>1$ then for each each forward arrow $(x, y) \in S$ must satisfy $x \leq k-1$ and $y \geq k$. (Forward arrows must nest $(k-1, k)$.)

(5) No head of an arrow in the set $S$ is also the tail of another arrow in $S$.

(6) No two arrows cross. 
Proof. Condition (1) is equivalent to Lemma 2.1. Except for Condition (3), the remaining conditions are stated for all faces in Proposition 5.5. To prove Condition (3), observe that $k=1$ implies that the backward arrow $(n+1,1)$ belongs to $S$. This arrow would nest any forward arrow, contradicting Condition (3) in Proposition 5.5.

\section{Triangulating Cho's Decomposition}

The type $A$ root polytope $P_{n}^{+}$is the convex hull of the origin and the set of points $\left\{e_{i}-e_{j}: 1 \leq\right.$ $i<j \leq n+1\}$. Cho [7] gave a decomposition of the Legendre polytope $P_{n}$ into $n+1$ copies of $P_{n}^{+}$as follows. The symmetric group $\mathfrak{S}_{n+1}$ acts on the Euclidean space $\mathbb{R}^{n+1}$ by permuting the coordinates, that is, the permutation $\sigma \in \mathfrak{S}_{n+1}$ sends the basis vector $e_{i}$ into $e_{\sigma(i)}$. Hence the permutation $\sigma$ acts on the Legendre polytope $P_{n}$ by sending each $e_{i}-e_{j}$ into $e_{\sigma(i)}-e_{\sigma(j)}$. Cho's main result [7, Theorem 16] is the following decomposition.

Theorem 7.1 (Cho). The Legendre polytope $P_{n}$ has the decomposition

$$
P_{n}=\bigcup_{k=0}^{n} \zeta^{k}\left(P_{n}^{+}\right)
$$

where $\zeta$ is the cycle $(1,2, \ldots, n+1)$. Furthermore, for $0 \leq k<r \leq n$ the polytopes $\zeta^{k}\left(P_{n}^{+}\right)$and $\zeta^{r}\left(P_{n}^{+}\right)$ have disjoint interiors.

In this section we show that each copy $\zeta^{k}\left(P_{n}^{+}\right)$of $P_{n}^{+}$is the union of simplices of the triangulation given in Definition [5.1, representing the boundary complex $\Gamma_{n}^{B}$ of Simion's type $B$ associahedron.

Theorem 7.2. Every facet $F$ of the arc representation of $\Gamma_{n}^{B}$ given in Definition 5.1 is contained in $\zeta^{k-1}\left(P_{n}^{+}\right)$where $k$ is the unique arrow of the form $(k-1, k)$ in $F$ or $(n+1,1)$ if $k=1$. Equivalently, the facet $F$ is contained in $\zeta^{k}\left(P_{n}^{+}\right)$exactly when it represents a facet of $\Gamma_{n}^{B}$ that contains the diagonal $\{k, \bar{k}\}$.

Proof. The polytope $P_{n}^{+}$is the convex hull the origin and of all vertices that are represented by backward arrows. The facets of type 1 (as defined in Theorem 6.4) form a pulling triangulation of the part of the boundary of $P_{n}^{+}$that does not contain the origin. In fact, the restriction of the pulling order to the backward arrows may be taken in the revlex order, as defined in [13, Definition 4.5], giving rise to the standard triangulation described in [10]. A facet in our triangulation of $P_{n}$ belongs to the standard triangulation of the boundary of the part of $P_{n}^{+}$not containing the origin exactly when it has type 1.

Observe next that the effect of $\zeta$ on the arrows (considered as vertices of $P_{n}$ ) is adding 1 modulo $n+1$ to the head and to the tail of each arrow. Taking into account Definition 5.1 and Remark 5.3 . it is not difficult to see that the induced effect on the arc representation is adding 1 modulo $n+1$, that is, a rotation. It is worth noting that to rotate each vertex of a regular $(2 n+2)$-gon into itself requires increasing each index by one $2 n+2$ times. However, a centrally symmetric pair of arcs $\{[u, v-1],[u+n+1, v+n]\}$ is taken into itself already by $n+1$ such elementary rotations. In the arc representation the facets containing only backward arrows corresponding to facets containing the pair of arcs $\{[1, n+1],[n+2,2 n+2]\}$. The induced action of $\zeta^{k-1}$ takes this pair into $\{[k, n+k],[n+$ 
$k+1,2 n+1+k]\}$. The conditions stated in Theorem 4.3 are rotation-invariant, so the induced action of $\zeta^{k-1}$ takes the family of all facets containing $\{[1, n+1],[n+2,2 n+2]\}$ into the family of all facets containing $\{[k, n+k],[n+k+1,2 n+1+k]\}$.

\section{A bijection Between the faces of $\Gamma_{n}^{B}$ And Delannoy paths}

In Simion's original paper, the nontrivial computation of the $f$-vector of the type $B$ associahedron $\Gamma_{n}^{B}$ used the recursive structure of the $B$-diagonals. Simion asked if there is a direct way to obtain this $f$-vector. Due to the unimodularity of the Legendre polytope $P_{n}$, all pulling triangulations of the boundary give the same $f$-vector. A direct enumeration in the case of the lexicographic pulling order, also known as the anti-standard triangulation, is straightforward and well-known; see [1, 13].

In this section we establish a bijection between the faces of $\Gamma_{n}^{B}$ and balanced Delannoy paths of length $2 n$ in such a way that $(k-1)$-dimensional faces correspond to Delannoy paths with $k$ up steps, thus answering Simion's question.

Definition 8.1. A balanced Delannoy path of length $2 n$ is a lattice path starting at $(0,0)$, ending at $(2 n, 0)$ and using only steps of the following three types: up steps $(1,1)$, down steps $(1,-1)$ and horizontal steps $(2,0)$. A Schröder path is a balanced Delannoy path that never goes below the horizontal axis.

More generally, a Delannoy path is a path taking the steps $(1,1),(1,-1)$ and $(2,0)$ with no ending condition. In this paper we will only work with balanced Delannoy paths.

Denote the up, down and horizontal steps by $U, D$ and $H$, respectively. We say that the length of the letters $U$ and $D$ is 1 , whereas the length of the letter $H$ is 2. A Delannoy path is uniquely encoded by a word in these three letters. We call the associated word a Delannoy word. A balanced Delannoy path of length $2 n$ corresponds to a word in which the number $k$ of the occurrences of $U$ is the same as the number of occurrences of $D$, and the number of occurrences of $H$ is $n-k$. We call such a word balanced. Hence the number of Delannoy paths of length $n$ with $k$ up steps is given by the

multinomial coefficient $\left(\begin{array}{c}n+k \\ k, k, n-k\end{array}\right)=\left(\begin{array}{c}n+k \\ k\end{array}\right)\left(\begin{array}{l}n \\ k\end{array}\right)$ which is the same as the number of $(k-1)$-dimensional faces in any pulling triangulation of the boundary of $P_{n}$; see Lemma 2.7.

We represent the faces of $\Gamma_{n}^{B}$ as a digraph on the set of nodes $\{1,2, \ldots, n+1\}$. A contrapositive formulation of Proposition 5.5 is the following.

Proposition 8.2. A digraph represents a face of Simion's type $B$ associahedron $\Gamma_{n}^{B}$ exactly when the following conditions are satisfied:

(1) There are no crossings between arrows.

(2) Forward arrows nest.

(3) A backward arrow cannot nest a forward arrow.

(4) No head of an arrow is the tail of another arrow. 


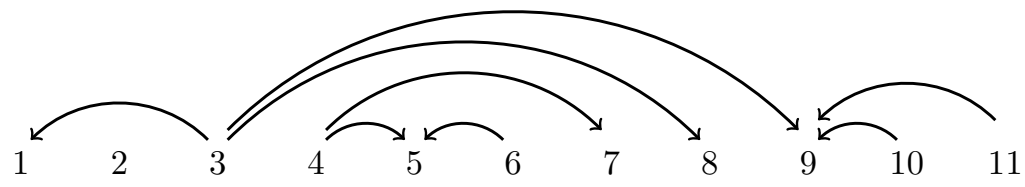

Figure 2. A valid digraph on 11 nodes.

See also Table 1 occurring in the proof of Theorem 6.1. Call such a set of arrows a valid digraph. An example of a valid digraph is shown in Figure 2.

For $W$ a subset of $V$ let $A_{W}$ denote the induced subgraph on $W$. Let $\alpha \cdot \beta$ denote the concatenation of the two words $\alpha$ and $\beta$.

Let $A$ be a valid digraph of backward arrows on a non-empty finite set of nodes $V \subseteq \mathbb{P}$ and let $v$ be the minimal element of $V$. We define the Schröder word $\operatorname{SP}(A)$ by the following recursive definition.

(i) If the set $V$ only consists of one node, that is, $V=\{v\}$, let $\operatorname{SP}(A)$ be the empty word $\epsilon$.

(ii) If the minimal node $v$ is an isolated node of $A$, let $\operatorname{SP}(A)$ be the concatenation $H \cdot \operatorname{SP}\left(A_{V-\{v\}}\right)$.

(iii) Lastly, when the node $v$ is not isolated then since $v$ is the least element, there is necessarily a backward arrow $(x, v)$ going into $v$. Let $w$ the smallest node with an arrow to $v$, that is, $w=\min (\{x \in V:(x, v) \in A\})$. Define $\mathrm{SP}(A)$ by

$$
\mathrm{SP}(A)=U \cdot \operatorname{SP}\left(A_{V \cap(v, w]}\right) \cdot D \cdot \operatorname{SP}\left(A_{V-(v, w]}\right) .
$$

Lemma 8.3. Let $A$ be a valid digraph on a set of nodes of size $n+1$ consisting of exactly $k$ backward arrows. The Schröder word $\mathrm{SP}(A)$ then has length $2 \cdot n$ and has exactly $k$ copies of the letters $U$ and $k$ copies of the letters $D$.

Proof. Both statements follow by induction on $n$. The first statement follows from the fact that the two sets of nodes $V \cap(v, w]$ and $V-(v, w]$ are complements of each other. The second statement follows by observing the noncrossing property ensures each arrow in $A$ is either the arrow $(w, v)$, an arrow in $A_{V \cap(v, w]}$, or an arrow in $A_{V-(v, w]}$.

Proposition 8.4. The map SP is a bijection between the set of all valid digraphs consisting only of backward arrows on the set of $n+1$ nodes and all Schröder words of length $2 n$.

Proof. Given a Schröder word $\alpha$ of length $2 n$ and a node set $V=\left\{v_{1}<v_{2}<\cdots<v_{n+1}\right\}$, the inverse map is computed recursively as follows. If $\alpha$ is the empty word $\epsilon$ then the inverse image is the isolated node $v_{1}$. If the word $\alpha$ begins with $H$, that is, $\alpha=H \cdot \beta$ where $\beta$ has length $2 n-2$, compute the inverse image of $\beta$ on the nodes $\left\{v_{2}, v_{3}, \ldots, v_{n+1}\right\}$ and add the node $v_{1}$ as an isolated node. Otherwise factor $\alpha$ uniquely as $U \cdot \beta \cdot D \cdot \gamma$, where $\beta$ and $\gamma$ are Schröder words of lengths $2 p$ and $2 n-2 p-2$, respectively. Compute the inverse image of $\beta$ on the nodes $\left\{v_{2}, v_{3}, \ldots, v_{p+2}\right\}$. Similarly, compute the inverse image of $\gamma$ on the nodes $\left\{v_{1}, v_{p+3}, v_{p+4}, \ldots, v_{n+1}\right\}$. Take the union of these two digraphs and add the backward arrow $\left(v_{p+2}, v_{1}\right)$. 
We now extend the bijection SP to all valid digraphs. In order to do this we introduce a twisting operation tw on each digraph $A$ that has a forward arrow from the least element $v \in V$ to the largest element $w \in V$. The twisted digraph $\operatorname{tw}(A)$ is a digraph on the node set $V-\{v\}$ with arrow set

$$
\operatorname{tw}(A)=A_{V-\{v\}} \cup\{(w, z):(v, z) \in A\} .
$$

In other words, we remove the least node $v$ and replace each forward arrow $(v, z)$ starting at $v$ with a backward arrow $(w, z)$. Note that there is no backward arrow $(z, v)$ in $A$ as $v$ is the tail of the forward arrow $(v, w)$.

Lemma 8.5. Let $V$ be a node set of smallest node $v$ and largest node $w$. The twisting map is a bijection from the set of valid digraphs on the set $V$ containing the forward arrow $(v, w)$ to the set of valid digraphs on the set $V-\{v\}$.

Proof. We claim that the twisted digraph $\operatorname{tw}(A)$ is a valid digraph. Begin to note that the restriction $A_{V-\{v\}}$ is a valid digraph. If there are no forward arrows in $A$ of the form $(v, z)$ where $z<w$, the equality $\operatorname{tw}(A)=A_{V-\{v\}}$ holds and the claim is true. Hence assume that there are forward arrows of the form $(v, z)$. We need to show that adding the backward arrow $(w, z)$ the digraph is still valid. We verify conditions (1) through (4) of Proposition 8.2 in order. If the arrow $(w, z)$ crosses an arrow $(x, y)$ then the arrow $(v, z)$ already crossed this arrow in $A$, verifying condition (1). Since we did not introduce any new forward arrows, condition (2) holds vacuously. Assume that the backward arrow $(w, z)$ nests a forward arrow $(x, y)$. Then the two forward arrows $(v, z)$ and $(x, y)$ did not nest in $A$, verifying (3). The last condition (4) holds directly, proving the claim.

The twisting map is one-to-one. Its inverse is given by

$$
\mathrm{tw}^{-1}(B)=\{(v, w)\} \cup B_{V \cap(v, w)} \cup\{(v, z):(w, z) \in B\} .
$$

Note that in case the node $w$ was a 'tail node' the inverse map switches it back to being a 'head node'.

We now extend the bijection SP to a map DP which applies to all valid digraphs.

(i) If the valid digraph $A$ has no forward arrows, let $\mathrm{DP}(A)=\operatorname{SP}(A)$.

(ii) If the valid digraph $A$ has a forward arrow, let $(x, y)$ be the forward arrow which nests the other forward arrows. Let $v$ and $w$ be the minimal, respectively, the maximal node of the node set $V$, that is, $v=\min (V)$ and $w=\max (V)$. Observe that $v \leq x<y \leq w$, that is, the two digraphs $A_{V \cap[v, x]}$ and $A_{V \cap[y, w]}$ have no forward arrows. Define $\operatorname{DP}(A)$ to be the concatenation

$$
\operatorname{DP}(A)=\operatorname{SP}\left(A_{V \cap[v, x]}\right) \cdot D \cdot \operatorname{DP}\left(\operatorname{tw}\left(A_{V \cap[x, y]}\right)\right) \cdot U \cdot \operatorname{SP}\left(A_{V \cap[y, w]}\right) .
$$

As an extension of Lemma 8.3 we have the following lemma.

Lemma 8.6. Let $A$ be a valid digraph on a set $V$ of $n+1$ nodes and assume that $A$ consists of $k$ arrows. Then the balanced Delannoy word $\mathrm{DP}(A)$ has length $2 n$ and has exactly $k$ copies of the letters $U$ and $D$, respectively. 


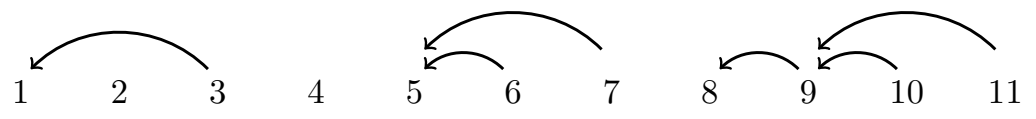

Figure 3. The modified set of arrows obtained from the set in Figure 2,

Proof. We only have to check the second case of the map DP. Assume that $V \cap[v, x]$ and $V \cap[y, w]$ have cardinalities $a$, respectively $b$. Then the middle part $V \cap[x, y]$ has size $n-a-b+3$. Thus $\operatorname{tw}\left(A_{V \cap[x, y]}\right)$ has $n-a-b+2$ nodes. Hence the total length is $2 \cdot(a-1)+2 \cdot(n-a-b+1)+2 \cdot(b-1)+2=2 n$. For the second statement it is again enough to check the second case. Assume that the restricted digraphs $A_{V \cap[v, x]}$ and $A_{V \cap[y, w]}$ have $c$, respectively $d$ arrows. Since there is no arrow nesting the forward arrow $(x, y)$, the middle digraph has $k-c-d$ arrows. Thus the twisted digraph has one less arrow, namely $k-c-d-1$. Hence the total number of $U$ letters is $c+(k-c-d-1)+d+1=k$, proving the second claim.

We now extend Proposition 8.4 to all valid digraphs.

Theorem 8.7. The map DP is a bijection between the set of all valid digraphs on a set of $n+1$ nodes and the set of all balanced Delannoy words of length $2 n$.

Proof. The inverse is computed as follows. Let $\alpha$ be a balanced Delannoy word of length $2 n$ and $V$ be a node set $\left\{v_{1}<v_{2}<\cdots<v_{n+1}\right\}$. If the Delannoy word $\alpha$ is a Schröder word, apply the inverse map of Proposition 8.4. Otherwise, we can factor the Delannoy word $\alpha$ uniquely as $\beta \cdot D \cdot \gamma \cdot U \cdot \delta$, where $\gamma$ is a balanced Delannoy word, and $\beta$ and $\delta$ are Schröder words. Note that the factor $\beta \cdot D$ is uniquely determined by the fact it is the shortest initial segment in which the number of $D$ letters exceeds the number of $U$ letters, in other words, the encoded path ends with the first down step going below the horizontal axis. By a symmetric argument, $U \cdot \delta$ is the shortest final segment with one more $U$ than the number of $D$ 's. Assume that $\beta, \gamma$ and $\delta$ have lengths $2 p, 2 q$ and $2 n-2 p-2 q-2$, respectively. Apply the inverse map from the proof of Proposition 8.4 to the words $\beta$ and $\delta$ to obtain digraphs on $\left\{v_{1}, v_{2}, \ldots, v_{p-1}\right\}$, respectively $\left\{v_{p+q+2}, v_{p+q+3}, \ldots, v_{n+1}\right\}$.

By recursion, apply the inverse of DP to $\gamma$ to obtain a valid digraph on the node set $\left\{v_{p+2}, v_{p+3}, \ldots\right.$, $\left.v_{p+q+2}\right\}$. Then apply the inverse of the twisting map to the digraph thus obtained. Finally, take the union of these three digraphs.

We now give non-recursive description of the bijection DP. First we encode a valid digraph with a multiset of indexed letters. Recall that a weakly connected component of a digraph is a connected component in the graph obtained by disregarding the direction of the arrows. In our valid digraphs, the weakly connected components are trees where each node is either a source or a sink.

Definition 8.8. Given a valid digraph $A$ on the node set $V$, define the associated multiset $M(A)$ with elements from the set of indexed letters $\left\{D_{x}, U_{x}, H_{x}: x \in \mathbb{P}\right\}$ by the following three steps:

(1) For each $x<\max (V)$ which is a maximum element in a weakly connected component we add the letter $H_{x}$ to the multiset $M(A)$. 


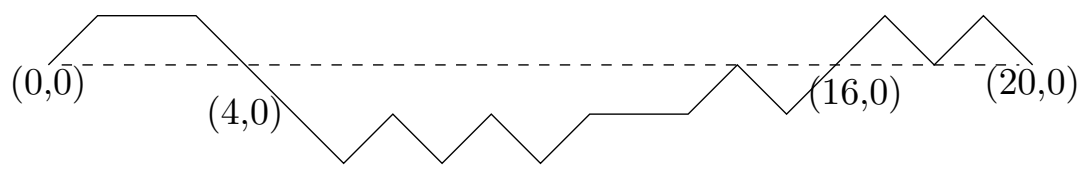

Figure 4. The lattice path associated to the digraph in Figure 2.

(2) For each tail $x$ of a forward arrow, consider the set $\operatorname{Head}(x)=\{y>x:(x, y) \in A\}$, that is, the set of heads of forward arrows with tail $x$. Add a copy of the letter $D_{x}$ to $M(A)$, also add a copy of the letter $U_{w}$ for $w=\max (\operatorname{Head}(x))$. Remove the forward arrow $(x, w)$. For each remaining $y \in \operatorname{Head}(x)$ that is less than $w$, replace the forward arrow $(x, y)$ with the backward arrow $(w, y)$. The resulting set of arrows has only backward arrows.

(3) For each head $y$ of a backward arrow, consider the set Tail $(y)=\{x>y:(x, y) \in A\}$, that is, the set of tails of backward arrows with head $y$. Add a copy of $U_{y}$ to $M(A)$. Also add a copy of $D_{x}$ for $x \in \operatorname{Tail}(y)$ and add a copy of $U_{x}$ for all but the maximum element of Tail $(y)$.

Example 8.9. For the valid set of arrows $A$ shown in Figure 2, in the first step we add the letters $H_{2}$ and $H_{7}$ to $M(A)$. In the second step we add the letters $D_{3}, U_{9}, D_{4}$, and $U_{7}$ to $M(A)$, and we remove the forward arrows $(3,9)$ and $(4,7)$. We also replace $(3,8)$ with $(9,8)$ and $(4,5)$ with $(7,5)$. We obtain the set of backward arrows shown in Figure 3. Note that this set of arrows does not need to be valid anymore: in our example, 9 is the head of $(10,9)$ and $(11,9)$ and it is also the tail of $(9,8)$. Finally, in step three we add the letters $U_{1}, D_{3}, U_{5}, D_{6}, U_{6}, D_{7}, U_{8}, D_{9}, U_{9}, D_{10}, U_{10}$, and $D_{11}$ to $M(A)$. We end up with the multiset

$$
M(A)=\left\{U_{1}, H_{2}, D_{3}, D_{3}, D_{4}, U_{5}, D_{6}, U_{6}, D_{7}, U_{7}, H_{7}, U_{8}, D_{9}, U_{9}, U_{9}, D_{10}, U_{10}, D_{11}\right\} .
$$

Define a linear order on the indexed letters by the inequalities $D_{x}<U_{x}<H_{x}<D_{x+1}$ for all positive integers $x$. We obtain the lattice path as follows.

Proposition 8.10. The balanced Delannoy word $\mathrm{DP}(A)$ is obtained from the multiset $M(A)$ by reading the indexed letters in order and then omitting the subscripts.

For the set of arrows shown in Figure 2, we obtain the word $U H D D D U D U D U H U D U U D U D$. The lattice path encoded by this word is shown in Figure 4.

A quick outline of a proof of Proposition 8.10 is as follows. First prove the statement when there are no forward arrows. In this case, each nonmaximal element $y$ of Tail $(x)$ contributes two consecutive letters $D_{y} U_{y}$. Next, observe that a digraph and and its twisted digraph have the same weakly connected components. Furthermore, the arrows that moved under the twisted operation still have the same set of heads. Hence, after recording the horizontal steps in step (1) we may perform all twisting operations simultaneously in step (2). We leave the remaining details to the reader.

\section{Concluding Remarks}

In recent paper, Cellini and Marietti [ [6] used abelian ideals to produce a triangulation for various root polytopes. In the case of type $A$, their construction yields once again a lexicographic triangulation 
of each face. Restricting to the positive roots yields Gelfand, Graev and Postnikov's anti-standard tree bases for the type $A$ positive root polytope. Is there an ideal corresponding to the reverse lexicographic triangulation?

The $h$-vector of Simion's type $B$ associahedron may be computed from the $f$-vector using elementary operations on binomial coefficients; see [20, Corollary 1].

Lemma 9.1 (Simion). The $h$-vector $\left(h_{0}, h_{1}, \ldots, h_{n}\right)$ of Simion's type $B$ associahedron $\Gamma_{n}^{B}$ satisfies

$$
h_{i}=\left(\begin{array}{c}
n \\
i
\end{array}\right)^{2} \quad \text { for } 0 \leq i \leq n \text {. }
$$

One would like to find a bijective proof of this result. One plausible way of attack would be to find an explicit shelling of $\Gamma_{n}^{B}$.

Finally, are there other interesting simplicial polytopes that can be better understood as pulling triangulations of less complicated polytopes?

\section{ACKNOWLEDGMENTS}

The first author was partially funded by the National Security Agency grant H98230-13-1-028. This work was partially supported by two grants from the Simons Foundation (\#245153 to Gábor Hetyei and \#206001 to Margaret Readdy). The authors thank the Princeton University Mathematics Department where this research was initiated.

\section{REFERENCES}

[1] F. Ardila, M. Beck, S. Hoşten, J. Pfeifle, K. Seashore, Root polytopes and growth series of root lattices, SIAM J. Discrete Math. 25 (2011), 360-378.

[2] C. Athanasiadis, $h^{*}$-vectors, Eulerian polynomials and stable polytopes of graphs, Electron. J. Combin. 11 (2) (2004), R6, 13 pp.

[3] R. Bott and C. Taubes, On the self-linking of knots. Topology and physics, J. Math. Phys. 35 (1994), $5247-5287$.

[4] H. Burgiel And V. Reiner, Two signed associahedra, New York J. Math. 4 (1998), 83-95 (electronic).

[5] C. Ceballos, F. Santos And G. M. Ziegler, Many non-equivalent realizations of the associahedron, Combinatorica 35 (2015), 513-551.

[6] P. Cellini and M. Marietti, Root polytopes and Abelian ideals, J. Algebr. Comb. 2014 (39), $607-645$.

[7] S. Chо, Polytopes of roots of type $A_{n}$, Bull. Austral. Math. Soc. 59 (1999), 391-402.

[8] E. Clark and R. Ehrenborg, Excedances of affine permutations, Adv. in Appl. Math. 46 (2011), $175-191$.

[9] R. Cori and G. Hetyei, Counting genus one partitions and permutations, Sém. Lothar. Combin. 70 (2013), Art. B70e, 29 pp.

[10] I. M. Gelfand, M. I. Graev, A. Postnikov, Combinatorics of hypergeometric functions associated with positive roots, in Arnold-Gelfand Mathematical Seminars: Geometry and Singularity Theory, Birkhäuser, Boston, 1996, 205-221.

[11] I. Heller, On linear systems with integral valued solutions, Pacific J. Math. 7 (1957), 1351-1364.

[12] I. Heller And A. J. Hoffman, On unimodular matrices, Pacific J. Math. 4 (1962), 1321-1327.

[13] G. Hetyei, Delannoy orthants of Legendre polytopes, Discrete Comput. Geom. 42 (2009), 705-721.

[14] J. F. P. Hudson, "Piecewise Linear Topology," W. A. Benjamin, Inc., New York and Amsterdam, 1969. 
[15] C. W. LEE, The associahedron triangulations of the n-gon, European J. Combin. 10 (1989), 551-560.

[16] M. MARKL, Simplex, associahedron, and cyclohedron. Higher homotopy structures in topology and mathematical physics (Poughkeepsie, NY, 1996), 235-265, Contemp. Math., 227, Amer. Math. Soc., Providence, RI, 1999.

[17] K. MÉszÁros, Root polytopes, triangulations, and the subdivision algebra, I, Trans. Am. Math. Soc. 363 (2011), 4359-4382.

[18] K. MÉszÁros, Root polytopes, triangulations, and the subdivision algebra, II, Trans. Am. Math. Soc. 363 (2011), 6111-6141.

[19] A. Postnikov, Permutohedra, associahedra, and beyond, Int. Math. Res. Not. IMRN (2009), $1026-1106$.

[20] R. Simion, A type-B associahedron, Adv. in Appl. Math. 30 (2003), 2-25.

[21] R. P. Stanley, Decompositions of rational convex polytopes, Ann. Discrete Math. 6 (1980), 333-342.

Department of Mathematics, University of Kentucky, Lexington, KY 40506-0027.

http://www.math.uky.edu/ jrge/, richard.ehrenborg@uky.edu .

Department of Mathematics and Statistics, UNC-Charlotte, Charlotte NC 28223-0001. http://www.math.uncc.edu/〜 ghetyei/, ghetyei@uncc.edu.

Department of Mathematics, University of Kentucky, Lexington, KY 40506-0027. http://www.math.uky.edu/〜readdy/, margaret.readdy@uky.edu . 